\title{
Riqueza e estrutura de uma comunidade vegetal de dunas na planície costeira de Santa Catarina'
}

\author{
João Carlos Ferreira Melo $\mathrm{Jr}^{2}$ \& Maria Regina Torres Boeger ${ }^{3}$
}

\author{
Parte da tese de doutorado do primeiro autor no Programa de Pós-Graduação em Ecologia e Conservação \\ da Universidade Federal do Paraná \\ ${ }^{2}$ Universidade da Região de Joinville, Laboratório de Anatomia e Ecologia Vegetal, Rua Paulo Maschitzki, 10, CEP 89219-710, \\ Joinville, Santa Catarina, Brasil. jcmelo_wood@hotmail.com \\ ${ }^{3}$ Universidade Federal do Paraná, Laboratório de Ecologia e Morfologia Funcional, Setor de Ciências Biológicas, \\ Caixa Postal 19031,CEP 81531-990, Curitiba, Paraná, Brasil.mboeger@ufpr.br
}

Recebido em 15.V.2015

Aceito em 24.X.2018

DOI 10.21826/2446-8231201873306

\begin{abstract}
RESUMO - Os estudos florísticos e estruturais sobre as restingas em dunas costeiras são ainda são incipientes. Este estudo avaliou a riqueza florística e a estrutura fitossociológica da vegetação herbácea sobre dunas da Praia Grande, Parque Estadual Acaraí (PEA), São Francisco do Sul, SC. O caminhamento expedito e o método de parcelas foram adotados para os levantamentos florístico e fitossociológico. Setenta e sete espécies pertencentes a 31 famílias de plantas foram identificadas. Asteraceae e Poaceae apresentaram o maior número de espécies. Os resultados indicaram alta riqueza específica, com destaque para as caméfitas e hemicriptófitas, que apresentam estratégias para a fixação de dunas e estabilização das areias na região pós-praia. A riqueza encontrada foi mais elevada do que para algumas outras restingas herbáceas no Estado de Santa Catarina, indicando que, apesar dos impactos antrópicos no PEA, a flora ali encontrada reflete o bom estado de conservação da área nesta unidade de conservação.
\end{abstract}

Palavras-chave: fitossociologia, florística, costa, restinga, herbáceas

ABSTRACT - Richness and structure of a dune plant community in the coastal plain of Santa Catarina. Floristic and structural studies about restinga vegetation on the dunes are still incipient. This study evaluated the floristic richness and phytosociological structure of the herbaceous vegetation of the dunes of Praia Grande, Acaraí State Park (ASP), São Francisco do Sul, SC. The assessment of the species composition and structure was carried out through walks and plots. Surveys resulted in 77 species belonging to 31 plants families. Asteraceae and Poaceae presented the highest number of species. The dune vegetation presented a high specific richness, especially the camephytes and hemicryptophytes, which show strategies for dune fixation and sand stabilization in the post-beach zone. Values of species richness were higher than some other values found for herbaceous restingas of Santa Catarina, indicating that, despite the human impacts in the ASP, its flora reflects the good conservation status of the area in this conservation unit.

Keywords: phytosociology, floristic survey, coastal, restinga, herbaceous

\section{INTRODUÇÃO}

A restinga é uma formação pioneira composta por um mosaico de comunidades florística e estruturalmente distintas (Waechter 1990). Ocorre nas planícies litorâneas, desde o Estado do Pará até o Rio Grande do Sul (CostaNeto et al. 2001), ao longo de cordões arenosos originados pelos depósitos arenosos do quaternário formados pelas regressões e transgressões marinhas e modelados pela ação dos ventos (Bigarella 2001, IBGE 2012).

As comunidades de restinga são marginais à floresta tropical atlântica e estão submetidas a condições ambientais extremas, como alta salinidade, deficiência de nutrientes no solo, elevada temperatura, luminosidade direta, ventos fortes frequentes e déficit hídrico, que tornam o ambiente estressante e limitante à vegetação (Waechter 1985, Scarano et al. 2001, Scarano 2002).
As formações herbáceas ocupam as primeiras porções de areia da faixa pós-praia. Essa faixa, formada por depósitos geralmente eólicos, é representada, em geral, pelas dunas fixas e móveis (Cordazzo et al. 2006). As dunas são compostas por areias finas e médias quartzosas ou por sedimentos síltico-argilosos, dispostas em elevações irregulares que na ocasião das marés e/ou ventos são deslocadas (Almeida \& Araújo 1997, Teixeira et al. 1986). As dunas móveis são frequentemente umedecidas pela ação das ondas e pelo spray marinho e são restritivas à instalação e ao crescimento da comunidade vegetal (Scarano 2002).

Portanto, a composição florística nas dunas litorâneas é determinada por uma combinação de fatores ambientais de origem oceânica e continental, em diferentes escalas (Alves et al. 2007). Essa vegetação encontra-se sob constante estresse devido à dinâmica desse ecossistema (Defeo et al. 2009). Além disso, o gradiente latitudinal ao longo da 
costa brasileira também exerce influência sobre o ambiente de dunas, tornando as comunidades vegetais características e peculiares ao longo do litoral (Lacerda \& Araujo 1987).

Estudos sobre a diversidade florística das restingas foram desenvolvidos ao longo dos $5.000 \mathrm{~km}$ de extensão do litoral brasileiro (Holzer et al. 2004). No entanto, para a Região Sul do país, predominam os levantamentos realizados nos estados do Paraná e Rio Grande do Sul (Menezes et al. 2015). Apesar de a faixa litorânea de Santa Catarina (SC) ser extensa, com 29 municípios litorâneos, este Estado possui o menor número de estudos de caracterização florística e estrutural da vegetação de dunas, com apenas nove municípios amostrados (Reitz 1961, Danilevicz et al. 1990, Castellani et al. 1995, Falkenberg 1999, Klein et al. 2007, Menezes et al. 2015). O estudo de maior amplitude foi feito pelo Inventário Florístico Florestal de Santa Catarina (Vibrans et al. 2012), mas sua amostragem restringiu-se a apenas quatro municípios (Korte et al. 2013).

Por outro lado, as ações antrópicas associadas ao crescimento das áreas urbanas no litoral continuam exercendo grande pressão sobre os ambientes de restinga, destruindo-os progressivamente. De forma geral, em SC a restinga é um dos ambientes mais degradados em função da expansão imobiliária, da deposição de lixo sobre a vegetação e do tráfego de veículos sobre as dunas (Rocha et al. 2003, Daniel 2006). Assim, o conhecimento sobre a ecologia de dunas é crucial para a preservação desse ambiente, o que torna necessária a ampliação de estudos sobre as comunidades herbáceas de restinga no litoral sul do Brasil.

O presente estudo caracterizou a composição e a estrutura da vegetação de dunas do maior remanescente de restinga do município de São Francisco do Sul, assim como a classificação das espécies quanto a formas de vida com o objetivo de melhor compreender a dinâmica dessa vegetação.

\section{MATERIAL E MÉTODOS}

O estudo foi realizado na restinga herbácea situada na Praia Grande, município de São Francisco do Sul, litoral nordeste de Santa Catarina (26 $6^{\circ} 16^{\prime} 55^{\prime}$ 'S $48^{\circ} 31^{\prime} 54^{\prime \prime} \mathrm{W}$, Fig. 1). O remanescente de restinga pertence ao Parque Estadual do Acaraí, unidade de conservação de proteção integral, com 6.667 ha, que possui a maior área contínua de restinga no Estado (FATMA 2008) e é classificada como prioridade extremamente alta para a conservação da biodiversidade (PROBIO 2003).

Segundo Köppen, o clima é do tipo Cfa (mesotérmico sem estação seca definida e com verões quentes), apresentando pluviosidade anual de $2.372 \mathrm{~mm}$ e temperatura média anual de $21,3^{\circ} \mathrm{C}$ (EPAGRI 2014). O solo é classificado como Neossolo Quartzarênico (EMBRAPA 2013), derivado de sedimentos eólicos e marinhos, cobrindo cerca de $15 \%$ da área total do parque (Oliveira \& Vieira 2008).

As dunas costeiras ocupam uma extensão de $10,5 \mathrm{~km}$ e organizam-se em dunas frontais, rampas de deflação em áreas não vegetadas, dunas transversais e dunas parabólicas. Ao norte da praia, as dunas alcançam até $27 \mathrm{~m}$ de altura, com a diminuição gradativa em altura na direção sul, quando se tornam indistintas (FATMA 2008). A vegetação herbácea é dominante nessa faixa e ocorre a partir da zona de maré até as dunas parabólicas.

Para o levantamento da flora utilizou-se o método de caminhamento (Filgueiras et al. 1994), onde apenas as angiospermas foram coletadas. O material botânico, coletado entre 2010 e 2011, foi herborizado, identificado e incorporado ao Herbário da Universidade de Joinville (JOI). As identificações taxonômicas foram realizadas por meio de comparações em herbários, literatura especializada e auxílio de especialistas. A lista de espécies seguiu APG IV (2016) e os nomes das espécies e dos respectivos autores a Lista de Espécies da Flora do Brasil (Flora do Brasil 2020). As espécies vegetais foram classificadas quanto a formas de vida segundo Moore \& Chapmann (1986). O registro do grau de ameaça das espécies baseou-se na lista vermelha do Centro Nacional de Conservação da Flora (CNCFLORA 2016).

Para a análise da estrutura foram utilizadas parcelas de $1 \mathrm{~m}^{2}$ (Munhoz \& Araújo 2011). Ao longo de um transecto paralelo à linha de costa, três pontos equidistantes entre si $(1,5 \mathrm{~km})$ foram demarcados. Em cada ponto, 100 parcelas foram delimitadas com intervalos regulares de $1 \mathrm{~m}$ entre si, totalizando $300 \mathrm{~m}^{2}$ de área amostral. A cobertura das espécies foi estimada na escala de Causton (1988), com cinco classes que avaliam em termos percentuais a projeção da parte aérea da planta na superfície da parcela, sendo posteriormente adotado o valor médio de cobertura. Para cada espécie calcularam-se os valores absolutos e relativos de frequência e cobertura e o valor de importância (MuellerDombois \& Ellenberg 1974).

\section{RESULTADOS E DISCUSSÃO}

No levantamento florístico, 77 espécies foram identificadas, distribuídas em 65 gêneros e 31 famílias (Quadro 1). As famílias com maior riqueza específica foram Poaceae (13 espécies), Asteraceae (12), Fabaceae (sete), Cyperaceae e Amaranthaceae (quatro cada). Essas famílias representaram $57,1 \%$ do total de espécies levantadas. Entre as espécies amostradas, cinco estão na lista vermelha da flora ameaçada no Brasil nas seguintes categorias: menos preocupante (3), quase ameaçada (1) e em perigo (1) (Quadro 1). Entre as exóticas naturalizadas, a única espécie observada foi Centela asiatica (Apiaceae).

Levantamentos de riqueza em dunas no litoral de SC (Tab. 1) indicam números de espécies muito variáveis (22 a 225 espécies), contudo em áreas amostrais igualmente variáveis. Tal abrangência parece relacionar-se a vários aspectos como: condições abióticas e de preservação local, a data de realização do levantamento, além do esforço amostral e critérios metodológicos de cada estudo. A combinação desses fatores parece ser determinante na 

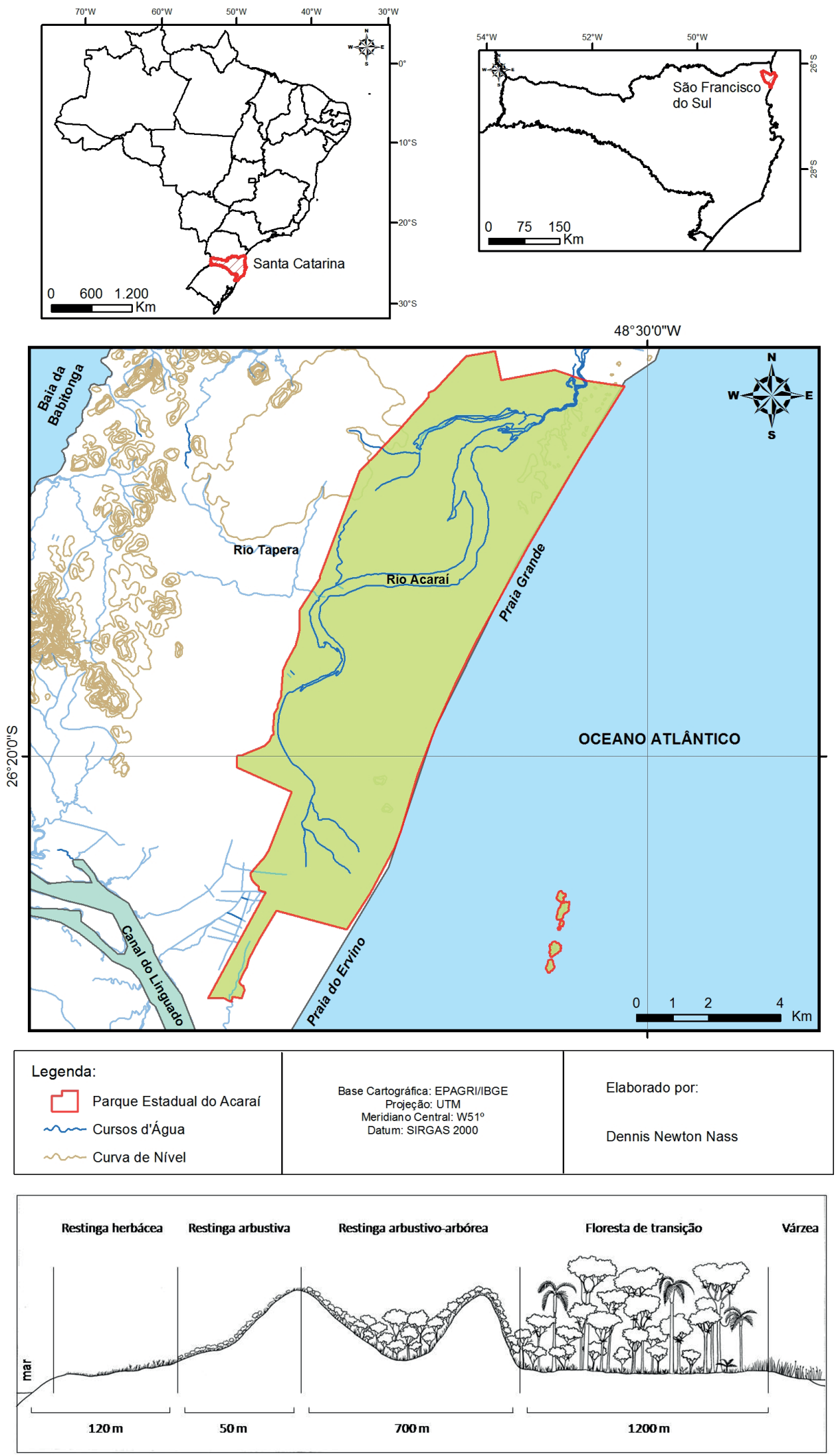

Fig. 1. Localização da restinga do Parque Estadual do Acaraí, São Francisco do Sul, Santa Catarina. 
Quadro 1. Relação das famílias e espécies encontradas na comunidade de dunas de restinga no Parque Estadual do Acaraí, São Francisco do Sul, SC. Categorias da IUCN: LC = menos preocupante, $\mathrm{NT}=$ quase ameaçada, $\mathrm{EN}=$ em perigo. $*$ espécie exótica naturalizada

\begin{tabular}{|c|c|c|}
\hline Família & Espécie (categoria de conservação segundo IUCN) & Forma de vida \\
\hline Aizoaceae & Sesuvium portulacastrum (L.) L. & caméfita \\
\hline \multirow[t]{4}{*}{ Amaranthaceae } & Alternanthera littoralis P. Beauv. & caméfita \\
\hline & Blutaparon portulacoides (A.St.-Hil.) Mears (LC) & caméfita \\
\hline & Chenopodium retusum (Moq.) Moq. & caméfita \\
\hline & Chenopodium sp. & caméfita \\
\hline \multirow[t]{2}{*}{ Apiaceae } & Centella asiatica (L.) Urb. * & geófita \\
\hline & Cyclospermum leptophyllum (Pers.) Britton P. Wilson & caméfita \\
\hline Apocynaceae & Oxypetalum tomentosum Wight ex Hook. \& Arn. & caméfita \\
\hline Araliaceae & Hydrocotyle bonariensis Lam. & geófita \\
\hline \multirow[t]{13}{*}{ Asteraceae } & Achyrocline alata DC. & caméfita \\
\hline & Achyrocline satureioides (Lam.) DC. & caméfita \\
\hline & Ageratum conyzoides $\mathrm{L}$. & caméfita \\
\hline & Baccharis crispa Spreng. & caméfita \\
\hline & Bidens alba (L.) DC. & caméfita \\
\hline & Conyza primulifolia (Lam.) Cuatrec. \& Lourteig & caméfita \\
\hline & Cyrtocymura scorpioides (Lam.) H. Rob. & caméfita \\
\hline & Gamochaeta americana (Mill.) Wedd. & caméfita \\
\hline & Hieracium commersonii Monnier & hemicriptófita \\
\hline & Noticastrum calvatum (Baker) Cuatrec. & caméfita \\
\hline & Pterocaulon lorentzii Malme & caméfita \\
\hline & Senecio crassiflorus (Poir) DC. (NT) & caméfita \\
\hline & Symphyopappus casarettoi B. L. Rob. & caméfita \\
\hline Boraginaceae & Varronia curassavica Jacq. & caméfita \\
\hline Bromeliaceae & Dyckia maritima Baker (EN) & hemicriptófita \\
\hline Cactaceae & Opuntia monacantha Haw. (LC) & caméfita \\
\hline \multirow[t]{2}{*}{ Calyceraceae } & Acicarpha bonariensis (Pers.) Herter & caméfita \\
\hline & Calycera crassifolia (Miers.) Hicken & caméfita \\
\hline Caryophyllaceae & Cardionema ramosissima (Weinm.) A. Nelson \& J. F. Macbr. & caméfita \\
\hline \multirow[t]{2}{*}{ Convolvulaceae } & Ipomoea imperati (Vahl) Griseb. & caméfita \\
\hline & Ipomoea pes-caprae (L.) R.Br. & caméfita \\
\hline \multirow[t]{4}{*}{ Cyperaceae } & Cyperus aggregatus (Willd.) Endl. & hemicriptófita \\
\hline & Cyperus ligularis $\mathrm{L}$. & hemicriptófita \\
\hline & Fimbristylis cymosa $\mathrm{R}$. Br. & hemicriptófita \\
\hline & Remirea maritima Aubl. & hemicriptófita \\
\hline \multirow[t]{2}{*}{ Euphorbiaceae } & Euphorbia hyssopifolia $\mathrm{L}$. & caméfita \\
\hline & Microstachys corniculata (Vahl) Griseb. & caméfita \\
\hline \multirow[t]{5}{*}{ Fabaceae } & Canavalia rosea (Sw.) DC. & caméfita \\
\hline & Centrosema virginianum (L.) Benth. & liana \\
\hline & Chamaecrista flexuosa (L.) Greene & caméfita \\
\hline & Desmodium adscendens (Sw.) DC. & caméfita \\
\hline & Desmodium barbatum (L.) Benth. & caméfita \\
\hline \multicolumn{3}{|c|}{ Quadro 1. Continuação } \\
\hline Família & Espécie (categoria de conservação segundo IUCN) & Forma de vida \\
\hline \multirow[t]{2}{*}{ Fabaceae } & Desmodium incanum DC. & caméfita \\
\hline & Stylosanthes viscosa (L.) Sw. & caméfita \\
\hline Goodeniaceae & Scaevola plumieri (L.) Vahl (LC) & caméfita \\
\hline Juncaceae & Juncus sp. & hemicriptófita \\
\hline
\end{tabular}


Quadro 1. Cont.

\begin{tabular}{|c|c|c|}
\hline Família & Espécie (categoria de conservação segundo IUCN) & Forma de vida \\
\hline \multirow[t]{3}{*}{ Malvaceae } & Pavonia sp. & caméfita \\
\hline & Sida carpinifolia $\mathrm{L}$. & caméfita \\
\hline & Waltheria indica $\mathrm{L}$. & caméfita \\
\hline Melastomataceae & Tibouchina clavata (Pers.) Wurdack & caméfita \\
\hline Menyanthaceae & Nymphoides indica (L.) Kuntze & caméfita \\
\hline Molluginaceae & Mollugo verticillata $\mathrm{L}$. & caméfita \\
\hline Orchidaceae & Epidendrum fulgens Brongn. & caméfita \\
\hline Orobanchaceae & Agalinis communis (Cham. \& Schltdl.) D’Arcy & caméfita \\
\hline Phyllantaceae & Phyllanthus niruri $\mathrm{L}$. & caméfita \\
\hline \multirow[t]{2}{*}{ Plantaginaceae } & Achetaria ocymoides (Cham. \& Schltdl.) Wettst. & caméfita \\
\hline & Plantago tomentosa Lam. & hemicriptófita \\
\hline \multirow[t]{13}{*}{ Poaceae } & Andropogon arenarius Hack. & hemicriptófita \\
\hline & Andropogon bicornis $\mathrm{L}$. & hemicriptófita \\
\hline & Cenchrus echinatus L. & hemicriptófita \\
\hline & Eragrostis trichocolea Arechav. & hemicriptófita \\
\hline & Eragrostis sp. & hemicriptófita \\
\hline & Eustachys retusa (Lag.) Kunth & hemicriptófita \\
\hline & Panicum racemosum (P. Beauv.) Spreng. & hemicriptófita \\
\hline & Panicum sp. & hemicriptófita \\
\hline & Paspalum pumilum Nees & hemicriptófita \\
\hline & Paspalum vaginatum $\mathrm{Sw}$. & hemicriptófita \\
\hline & Spartina ciliata Brongn. & hemicriptófita \\
\hline & Sporobolus virginicus (L.) Kunth & hemicriptófita \\
\hline & Stenotaphrum secundatum (Walter) Kuntze & hemicriptófita \\
\hline \multirow[t]{2}{*}{ Polygalaceae } & Polygala cyparissias A. St.-Hil. \&Moq. & caméfita \\
\hline & Polygala paniculata $\mathrm{L}$. & caméfita \\
\hline Portulacaceae & Portulaca oleracea $\mathrm{L}$. & caméfita \\
\hline \multirow[t]{3}{*}{ Rubiaceae } & Diodella apiculata (Willd. ex Roem. \&Schult.) Delprete & caméfita \\
\hline & Diodella radula (Willd. ex Roem. \&Schult.) Delprete & caméfita \\
\hline & Richardia brasiliensis Gomes & caméfita \\
\hline Sapindaceae & Dodonaea viscosa Jacq. & caméfita \\
\hline Smilacaceae & Smilax campestris Griseb. & caméfita \\
\hline
\end{tabular}

riqueza específica, pois áreas de dunas mais preservadas e protegidas de ventos tendem a ter maior riqueza de espécies (Araújo et al. 2016).

Os dados obtidos neste estudo corroboram outros levantamentos florísticos em área de restinga no litoral de $\mathrm{SC}$, os quais destacaram Asteraceae (13 espécies) e Poaceae (13 espécies) como as famílias de maior riqueza específica (Danilevicz et al. 1990, Souza et al. 1992, Daniel 2006, Guimarães 2006, Klein et al. 2007). Apenas o estudo de Castellani et al. (1995) destacou Poaceae e Cyperaceae como as famílias mais diversas, com Asteraceae em terceiro lugar.

A maior riqueza de espécies de Poaceae e Asteraceae se deve aos atributos ligados diretamente ao sucesso de crescimento, sobrevivência e reprodução das plantas. Características como suculência, pubescência, hábito prostrado, esclerofilia, metabolismo $\mathrm{C}_{4}$, reprodução vegetativa, alta produção flores e sementes, polinização anemófila, entre outros, permitem a manutenção dos processos metabólicos vitais (Barbour 1992) e, consequentemente, o desenvolvimento dessas plantas em ambientes inconsolados e inférteis, como as dunas.

Quanto às formas de vida, entre as espécies encontradas as caméfitas foram predominantes $(68,8 \%)$, seguida das hemicriptófitas $(27,2 \%)$. Ambas apresentam caules formados por longos estolões ou rizomas de crescimento horizontal rápido e finas raízes adventícias, podendo cobrir uma extensa área do solo (Pfadenhauer 1978, IBGE 2012). Os atributos dessas formas de vida podem ser considerados estratégias adaptativas que minimizam as condições estressantes (e.g., substrato instável, escassez de água, as altas temperaturas e a elevada salinidade), como ocorrem nas dunas (Seeliger 1992, Spanou et al. 2006, Araújo et al. 2016). Espécies como Blutaparon portulacoides, Cakile maritima, Canavalia rosea, Ipomoea pes-caprae, Paspalum vaginatum e Spartina ciliata, que apresentam tais atributos, são consideradas fixadoras de dunas, contribuindo 
Tabela 1. Riqueza de espécies e famílias mais diversas registradas em estudos realizados em ambiente de dunas em Santa Catarina, Brasil.

\begin{tabular}{|c|c|c|c|c|}
\hline Estudo & Município(s) & $\begin{array}{l}\text { Área amostral } \\
\qquad\left(\mathrm{m}^{2}\right)\end{array}$ & Espécies & Famílias com $>$ riqueza \\
\hline Presente estudo & São Francisco do Sul & 300 & 77 & $\begin{array}{c}\text { Poaceae (13) } \\
\text { Asteraceae (12) }\end{array}$ \\
\hline Reitz (1954) & Laguna & - & 176 & $\begin{array}{l}\text { Cyperaceae (11) } \\
\text { Myrtaceae (11) }\end{array}$ \\
\hline Bresolin (1979) & Florianópolis & - & 22 & $\begin{array}{c}\text { Poaceae (6) } \\
\text { Asteraceae (2) }\end{array}$ \\
\hline Cordazzo \& Costa (1989) & Garopaba & 130 & 31 & $\begin{array}{c}\text { Asteraceae (6) } \\
\text { Poaceae (4) }\end{array}$ \\
\hline Danilevicz et al. (1990) & Garopaba & 50 & 75 & $\begin{array}{c}\text { Asteraceae (16) } \\
\text { Poaceae (15) }\end{array}$ \\
\hline Souza et al. (1992) & Florianópolis & - & 112 & $\begin{array}{c}\text { Asteraceae }(29) \\
\text { Poaceae }(27)\end{array}$ \\
\hline Castellani et al. (1995) & Florianópolis & 75 & 61 & $\begin{array}{c}\text { Poaceae (15) } \\
\text { Cyperaceae (13) }\end{array}$ \\
\hline Daniel $(2006) *$ & Araranguá & 102 & 124 & $\begin{array}{c}\text { Poaceae (34) } \\
\text { Asteraceae (21) }\end{array}$ \\
\hline Guimarães (2006)* & Florianópolis & - & 104 & $\begin{array}{c}\text { Asteraceae (24) } \\
\text { Poaceae (12) }\end{array}$ \\
\hline Klein et al. (2007) & Araranguá & 120 & 60 & $\begin{array}{c}\text { Asteraceae (11) } \\
\text { Poaceae (9) }\end{array}$ \\
\hline Korte et al. $(2013)^{*, * *}$ & Araranguá, Florianópolis, Palhoça e São Francisco do Sul & 2.000 & 225 & $\begin{array}{l}\text { Asteraceae (22) } \\
\text { Cyperaceae (9) }\end{array}$ \\
\hline
\end{tabular}

*Dados restritos ao ambiente de dunas. **Não distinguem as formações de restinga onde foram encontradas as espécies herbáceas/subarbustivas identificadas.

para a estabilização dos cordões arenosos dispostos na região pós-praia (Cordazzo et al. 2006). Algumas espécies de Paspalum e Senecio crassiflorus são conhecidas por alcançarem maiores densidades em áreas de declive e expostas ao vento, aproveitando o aporte adicional de nutrientes trazido pelos ventos fortes (Pfadenhauer 1978).

O levantamento fitossociológico registrou 29 espécies, distribuídas em 28 gêneros e 16 famílias (Tab. 2). Entre as espécies mais frequentes destacaram-se Remirea maritima, Smilax campestris, Ipomoea imperati e Blutaparon portulacoides. A cobertura total da comunidade herbácea sobre dunas foi de aproximadamente 58\% da área amostrada, mas a maior parte das espécies (22) apresentou valores de cobertura inferiores a 3\%. A espécie com maior valor de cobertura foi Scaevola plumieri. As espécies com os maiores valores de importância (VI) foram Smilax campestris, Remirea maritima, Scaevola plumieri, Ipomoea imperati, Canavalia rosea, Blutaparon portulacoides, Ipomoea pes-caprae, Stylosanthes viscosa, Sesuvium portulacastrum e Oxypetalum tomentosum. O pequeno número de espécies (sete) com alto valor de importância $(>10)$, em relação ao número total de espécies identificadas (29), o que representa apenas $24 \%$, se deve aos altos valores de frequência e cobertura dessas espécies.

Resultados similares foram obtidos em outros estudos para SC, com pequenas variações de ocorrência de espécies (Bresolin 1979, Danilevicz et al. 1990, Castellani et al. 1995, Guimarães 2006, Klein et al. 2007). Apesar de assumirem diferentes posições sociológicas, as espécies ocorrentes nas dunas são, em sua maioria, as mesmas e possuem importante papel na colonização e estabilização das mesmas. É importante salientar que, apesar de um número expressivo de espécies vegetais se desenvolverem sobre as dunas (Tab. 2), são poucas as espécies que apresentam condições adaptativas para colonizar e formar densos agrupamentos sobre elas. Adaptações estruturais e fisiológicas são fundamentais para a manutenção dessas populações e várias são reportadas na literatura, como suculência, pubescência, hábito prostrado, metabolismo CAM, órgãos de reserva, polinização e dispersão pelo vento, entre outros (García-Mora et al. 1999, Boeger \& Gluzezak 2006, Rosado \& Mattos 2007). Uma vez estabelecidas as populações fixadoras de dunas, o ambiente torna-se mais propício para a colonização de novas espécies, permitindo assim um aumento na riqueza específica (Almeida \& Araújo, 1997).

Os resultados indicam que a vegetação apresentou alta riqueza específica, com destaque para as caméfitas e hemicriptófitas, que apresentam estratégias adaptativas para a fixação de dunas e estabilização das areias na região póspraia. Essas espécies têm importante papel na comunidade, pois preparam o ambiente para a colonização de novas espécies, aumentando assim a diversidade herbácea. $\mathrm{O}$ valor de riqueza específica deste estudo é superior ao registrado para algumas outras restingas herbáceas de SC, indicando que apesar dos impactos antrópicos no Parque do Estadual do Acaraí, pela coleta seletiva de espécimes para floricultura, pisoteio em certos trechos de dunas parabólicas 
Tabela 2. Espécies amostradas na comunidade de dunas da restinga do Parque Estadual do Acaraí, São Francisco do Sul, Santa Catarina, Brasil, em ordem decrescente de valor de importância. Legenda: Número de parcelas de ocorrência da espécie i (Npi), frequência absoluta (FA), frequência relativa (FR), cobertura absoluta (CA), cobertura relativa (CR) e valor de importância (VI).

\begin{tabular}{|c|c|c|c|c|c|c|c|}
\hline Família & Espécie & Npi & FA & FR & $\mathrm{CA}$ & CR & VI \\
\hline Smilacaceae & Smilax campestres & 212 & 70,67 & 17,24 & 14,09 & 7,98 & 25,22 \\
\hline Cyperaceae & Remirea maritima & 217 & 72,33 & 17,64 & 5,75 & 3,26 & 20,90 \\
\hline Goodeniaceae & Scaevola plumieri & 72 & 24,00 & 5,85 & 22,99 & 13,02 & 18,88 \\
\hline Convolvulaceae & Ipomoea imperati & 166 & 55,33 & 13,50 & 3,64 & 2,07 & 15,56 \\
\hline Fabaceae & Canavalia rosea & 79 & 26,33 & 6,42 & 13,70 & 7,76 & 14,19 \\
\hline Amaranthaceae & Blutaparon portulacoides & 122 & 40,67 & 9,92 & 5,45 & 3,09 & 13,01 \\
\hline Convolvulaceae & Ipomoea pes-caprae & 57 & 19,00 & 4,63 & 9,91 & 5,62 & 10,25 \\
\hline Fabaceae & Stylosantes viscosa & 33 & 11,00 & 2,68 & 7,88 & 4,46 & 7,15 \\
\hline Aizoaceae & Sesuvium portulacastrum & 6 & 2,00 & 0,49 & 10,83 & 6,14 & 6,63 \\
\hline Apocynaceae & Oxypetalum tomentosum & 50 & 16,67 & 4,07 & 4,40 & 2,49 & 6,56 \\
\hline Poaceae & Paspalum vaginatum & 48 & 16,00 & 3,90 & 4,58 & 2,60 & 6,50 \\
\hline Cactaceae & Opuntia monacantha & 4 & 1,33 & 0,33 & 10,63 & 6,02 & 6,35 \\
\hline Poaceae & Spartina ciliata & 49 & 16,33 & 3,98 & 3,93 & 2,23 & 6,21 \\
\hline Calyceraceae & Acicarpha spathulata & 2 & 0,67 & 0,16 & 10,00 & 5,67 & 5,83 \\
\hline Asteraceae & Symphyopappus casarettoi & 2 & 0,67 & 0,16 & 6,25 & 3,54 & 3,70 \\
\hline Malvaceae & Pavonia sp. & 2 & 0,67 & 0,16 & 6,25 & 3,54 & 3,70 \\
\hline Calyceraceae & Calycera crassifolia & 8 & 2,67 & 0,65 & 5,31 & 3,01 & 3,66 \\
\hline Asteraceae & Gamochaeta americana & 25 & 8,33 & 2,03 & 2,50 & 1,42 & 3,45 \\
\hline Apiaceae & Hydrocotyle bonariensis & 22 & 7,33 & 1,79 & 2,50 & 1,42 & 3,21 \\
\hline Apiaceae & Centella asiatica & 19 & 6,33 & 1,54 & 2,89 & 1,64 & 3,18 \\
\hline Asteraceae & Conyza primulifolia & 15 & 5,00 & 1,22 & 3,00 & 1,70 & 2,92 \\
\hline Amaranthaceae & Alternanthera littoralis & 10 & 3,33 & 0,81 & 2,50 & 1,42 & 2,23 \\
\hline Portulacaceae & Portulaca oleracea & 3 & 1,00 & 0,24 & 2,50 & 1,42 & 1,66 \\
\hline Fabaceae & Desmodium adscendens & 2 & 0,67 & 0,16 & 2,50 & 1,42 & 1,58 \\
\hline Fabaceae & Centrosema virginianum & 1 & 0,33 & 0,08 & 2,50 & 1,42 & 1,50 \\
\hline Euphorbiaceae & Euphorbia hyssopifolia & 1 & 0,33 & 0,08 & 2,50 & 1,42 & 1,50 \\
\hline Asteraceae & Pterocaulon purpurascens & 1 & 0,33 & 0,08 & 2,50 & 1,42 & 1,50 \\
\hline Malvaceae & Sida carpinifolia & 1 & 0,33 & 0,08 & 2,50 & 1,42 & 1,50 \\
\hline Poaceae & Stenotaphrum secundatum & 1 & 0,33 & 0,08 & 2,50 & 1,42 & 1,50 \\
\hline
\end{tabular}

e a própria estrada que corta a porção frontal da restinga, a flora ali encontrada reflete o bom estado de conservação da área pela consolidação da unidade de conservação.

\section{AGRADECIMENTOS}

Aos sistematas Dr. Osmar dos Santos Ribas do Museu Botânico Municipal de Curitiba e Dr. Rafael Trevisan da Universidade Federal de Santa Catarina pela identificação das espécies coletadas na restinga do Parque Estadual do Acaraí.

\section{REFERÊNCIAS}

Almeida, A.L. \& Araújo, D.S.D. 1997. Comunidades vegetais do cordão arenoso externo da Reserva Ecológica Estadual de Jacarepiá, Saquarema, RJ. Oecologia Brasiliensis 51:47-63.

Alves, R.J.V., Cardin, L. \& Kropf, M.S. 2007. Angiosperm disjunction "Campos rupestres - restingas": a re-avaluation. Acta Botanica Brasilica 21(3):675-685.
Angiosperm Phylogeny Group - APG IV. 2016. An update of the Angiosperm Phylogeny Group classification for the orders and families of flowering plants: APG IV. Biological Journal of the Linnean Society 181:1-20.

Araújo, A. C. M., da Silva, A.N.F., de Almeida Jr., E.B. 2016. Caracterização estrutural e status de conservação do estrato herbáceo de dunas da Praia de São Marcos, Maranhão, Brasil. Acta Amazônica 46: 247 - 258.

Barbour, M.G. 1992. Life at the Leading Edge: The Beach Plant Syndrome. In Seeliger, U. (Ed.): Coastal Plant Communities of Latin America. Academic Press, San Diego, p. 291-307.

Bigarella, J.J. 2001. Contribuição ao estudo da planície litorânea do estado do Paraná. Brazilian Archives of Biology and Technology 65-110.

Boeger, M.R.T. \& Gluzezak, R.M. 2006. Adaptações estruturais de sete espécies de plantas para as condições ambientais da área de dunas de Santa Catarina, Brasil. Iheringia, Série Botânica 61(1-2):73-82.

Bresolin, A. 1979. Flora da restinga da Ilha de Santa Catarina. Insula 10:1-55.

Castellani, T.T., Folchini, R. \& Scherer, K.Z. 1995. Variação temporal da vegetação em um trecho de baixada úmida entre dunas, Praia da Joaquina, Florianópolis, SC. Insula 24:37-72.

Causton, D.R. 1988. Introduction to vegetation analysis. Unwin Hyman, London. 342 p. 
Centro Nacional de Conservação da Flora - CNCFLORA. 2016. Lista vermelha. Disponível em: http://cncflora.jbrj.gov.br/portal/pt-br/ listavermelha. Acessado em 27.06.2016.

Cordazzo, C.V. \& Costa, C.S.B. 1989. Associações vegetais das dunas frontais de Garopaba (SC). Ciência e Cultura 41(9):906-910.

Cordazzo, C.V., Paiva, J.B. \& Seeliger, U. 2006. Plantas de dunas da costa sudeste atlântica. União Sulamaricana de Estudos da Biodiversidade, Pelotas. $107 \mathrm{p}$.

Costa-Neto, S.V., Pereira, O.J., Bastos, M.N.C., Santos, J.U.M. \& Amaral, D.D. 2001. Fitossociologia das formações herbáceas da restinga do Crispim, Marapanim - PA. Boletim do Museu Paraense Emílio Goeldi. Série Botânica 17(1):161-185.

Daniel, R.B. 2006. Florística e fitossociologia da restinga herbáceaarbustiva do Morro dos Conventos, Araranguá-SC. Dissertação, 74 f., Universidade do Extremo Sul Catarinense, Criciúma.

Danilevicz, E., Janke, H. \& Pankowski, L.H.S. 1990. Florística e estrutura da comunidade herbácea e arbustiva da Praia do Ferrugem, Garopaba, SC. Acta Botanica Brasilica 4(2):21-34.

Defeo, O., McLachlan, A., Schoeman, D.S., Schlacher, T.A., Dugan, J., Jones, A., Lastra, M. \& Scapini, F. 2009. Threats to sand beach ecosystems: a review. Estuarine, Costal and Shelf Science 81:1-12.

Empresa Brasileira de Pesquisa Agropecuária - EMBRAPA. 2013. Sistema brasileiro de classificação de solos. Empresa Brasileira de Pesquisa Agropecuária, Brasília. 353 p.

Empresa de Pesquisa Agropecuária e Extensão Rural de Santa Catarina EPAGRI. 2014. Dados meteorológicos. Disponível em: http://www. epagri.sc.gov.br. Acessado em 10.03.2014.

Falkenberg, D.B. 1999. Aspectos da flora e da vegetação secundária da restinga de Santa Catarina, sul do Brasil. Insula 28:1-30.

Fundação Estadual do Meio Ambiente - FATMA. 2008. Plano de manejo do Parque Estadual do Acaraí. FATMA, Curitiba. 240 p.

Filgueiras, T.S., Nogueira, P.E., Brochado, A.L. \& Guala II, G.F. 1994. Caminhamento: um método expedito para levantamentos florísticos qualitativos. Cadernos de Geociências, Instituto Brasileiro de Geografia e Estatística 12:39-43.

García-Mora, M.R., Gallego-Fernandez, J.B. \& García-Novo, F. 1999. Plant funcional types in coastal foredunes in relation to environmental stress and disturbance. Journal of Vegetation Science 10:27-34.

Guimarães, T.B. 2006. Florística e fenologia reprodutiva de plantas vasculares na restinga do Parque Municipal das Dunas da Lagoa da Conceição, Florianópolis, SC. Dissertação, 107 f., Universidade Federal de Santa Catarina, Florianópolis.

Holzer, W., Crichyno, J. \& Pires, A.C. 2004. Sustentabilidade da urbanização em áreas de restinga: uma proposta de avaliação pósocupação. Paisagem Ambiente 19:49-66.

Instituto Brasileiro de Geografia e Estatística - IBGE. 2012. Manual técnico da vegetação brasileira. IBGE, Rio de Janeiro. 274 p.

Klein, A.S., Citadini-Zanette, V. \& Santos, R. 2007. Florística e estrutura comunitária de restinga herbácea no município de Araranguá, Santa Catarina. Biotemas 20(3):15-26.

Korte, A., Gasper, A.L., Kruger, A. \& Sevegnani, L. 2013. Composição florística e estrutura das restingas de Santa Catarina. In: Inventário florísico florestal de Santa Catarina: floresta ombrófila densa (A. C. Vibrans et al., eds.). Editora da Fundação Universitária de Blumenau, Blumenau, pp. 285-309.

Lacerda, L.D. \& Araujo, D.S.D. 1987. A natureza das restingas. Ciência Hoje 6(33):42-49.

Menezes, L.S., Müller, S.C. \& Overbeck, G.E. 2015. Floristic and structural patterns in South Brazilian coastal grasslands. Anais da Academica Brasileira de Ciências 87: 2081-2090.

Moore, P.D. \& Chapmann, S.B. 1986. Methods in plant ecology. Blackwell Scientific Publications, Oxford. 598 p.

Mueller-Dombois, D. \& Ellenberg, H. 1974. Aims and methods of vegetation ecology. John Wiley and Sons, New York .547 p.
Munhoz, C.B.R. \& Araújo, G.M. 2011. Métodos de amostragem do estrato herbáceo-subarbustivo. In Felfili, J.M, Eisenlohr, P.V., Melo, M.M.R.F, Andrade, L.A. \& Neto, J.A.A.M. (eds.). Fitossociologia no Brasil. Universidade Fedral de Viçosa, Viçosa. p. 213-232.

Oliveira, F.A. \& Vieira, C.V. 2008. Proposal of semi-detailed geomorphological map of northeastern Santa Catarina State, Brazil. In Abstract book of the international meeting and field trip environmental analysis and geomorphological mapping for a sustainable development. Roma University, Roma. p. 13-15.

Pfadenhauer, J. 1978. Contribuição ao conhecimento da vegetação e de suas condições de crescimento das dunas costeiras do Rio grande do Sul, Brasil. Revista Brasileira de Biologia 38(4):827-836.

Projeto de Conservação e Utilização Sustentável da Diversidade Biológica Brasileira - PROBIO. 2003. Áreas prioritárias para a conservação, utilização sustentável e repartição de benefícios da biodiversidade brasileira. Ministério do Meio Ambiente, Brasília. 13p.

Reitz, R. 1954. A vegetação de Laguna. Sellowia 6(6):243-258.

Reitz, R. 1961. Vegetação da zona marítima de Santa Catarina. Sellowia 13(13):17-111.

Rocha, C.F.D., Bergallo, H.G., Alves, M.A.S. \& Van Sluys, M. 2003. A biodiversidade nos grandes remanescentes florestais do estado do Rio de Janeiro e nas restingas da Mata Atlântica. RiMa, São Carlos. 160 p.

Rosado, B.H.P. \& Mattos, E. A. de. 2007. Variação temporal de características morfológicas de folhas em dez espécies do Parque Nacional da restinga de Jurubatiba, Macaé, RJ, Brasil. Acta Botanica Brasilica 21:741-752.

Scarano, F.R. 2002. Structure, function and floristic relationships of plant communities in stressful habitats to the Brazilian atlantic rainforest. Annals of Botany 90:517-524.

Scarano, F.R., Duarte, H.M., Ribeiro, K.T., Rodrigues, P.J.F.P., Barcellos, E.M.B., Franco, A.C., Brulfert, J., DeleÂens, E. \& Luèttge, U. 2001. Four sites with contrasting environmental stress in southeastern Brazil: relations of species, life form diversity, and geographical distribution to ecophysiological parameters. Botanical Journal of the Linnean Society 136:345-364.

Seeliger, U. 1992. Coastal dunes of southern Brazil: physiography, habitats and vegetation. In Subtropical convergence environments: the coastal sea in the southwestern Atlantic (Seeliger, U., Odebrecht, C., \& Castello, J.P., eds.). Springer-Verlag, Berlin. p. 367-381.

Souza, M.L.D.R., Falkenberg, D.B., Amaral, L.G., Fronza, M., Araújo, A.C. \& Sá, M.R. 1992. Vegetação do Pontal da Daniela, Florianópolis, SC, Brasil. I. Levantamento florístico e mapa fitogeográfico. Insula 21:87-117.

Spanou, S., Verroios, G., Dimitrellos, G., Tiniakou, A. \& Georgiadis, T. 2006. Notes on flora and vegetation of the sand dunes of western Greece. Willdenowia 36:235-246.

Teixeira, M.B., Coura-Neto, A.B., Pastore, U. \& Rangel-Filho, A.L.R. 1986. As regiões fitoecológicas, sua natureza e seus recursos econômicos - Estudo fitogeográfico. In Instituto Brasileiro de Geografia e Estatística. Levantamento de recursos naturais. IBGE, Rio de Janeiro. p. 541-620.

Vibrans, A.C., Sevegnani, L., Gasper, A.L. \& Lingner, D.V. 2012. Inventário Florístico Florestal de Santa Catarina: diversidade e conservação dos remanescentes florestais. Edifurb, Blumenau. 334 p.

Waechter, J.L. 1985. Aspectos ecológicos da vegetação da restinga no Rio Grande do Sul, Brasil. Comunicações do Museu de Ciências da Pontifícia Universidade Católica do Rio Grande do Sul. Série Botânica 33:49-68.

Waechter, J.L. 1990. Comunidades vegetais das restingas do Rio Grande do Sul. In Anais do II Simpósio de Ecossistemas da Costa Sul e Sudeste Brasileira. Academia de Ciência do Estado de São Paulo, Águas de Lindóia, SP, p. 228-248.

Zanella, N.R.Z, Prudencio, M. \& Castellani, T.T. 2010. Análise da cobertura vegetal em duna semifixa dez anos após a aplicação de técnicas de restauração no Parque Municipal das Dunas da Conceição, Florianópolis, Santa Catarina. Biotemas 23(3):49-58. 